\title{
CIRURGIA AMBULATORIAL EM PROCTOLOGIA: análise retrospectiva de 437 casos
}

\author{
Rogério SAAD-HOSSNE, Renê Gamberini PRADO e Alexandre BAKONYI-NETO
}

RESUMO - Racional - O número de cirurgias ambulatoriais realizadas em hospitais, como em clínicas particulares, cresce a cada dia. Hoje, em alguns países, como a França, há predomínio das cirurgias ambulatoriais em relação às hospitalares. Objetivo - Avaliar retrospectivamente os pacientes operados no Serviço de Cirurgia Ambulatorial do Hospital das Clínicas da Faculdade de Medicina de Botucatu, SP. Casuística e Método - Foram estudados retrospectivamente 437 casos clínicos de patologias orificiais, analisando a distribuição por faixa etária, por sexo, por patologias e as complicações pós-operatórias. Resultado - Notou-se predomínio de pacientes com idade inferior a 45 anos (62,8\%), prevalência do sexo feminino (56\%), sendo a doença hemorroidária $(45,1 \%)$ a principal patologia e a dor e sangramento as complicações mais freqüentes $(9,8 \%$ e 7,3\%). Conclusĩes - Os resultados satisfatórios observados demonstram a possibilidade de realização, em ambulatório, de diversos procedimentos simples em patologias anorretais freqüentes, a baixo custo e poucas complicações, sendo estas não superiores às observadas em cirurgia hospitalar.

DESCRITORES - Intestino grosso, cirurgia. Procedimentos cirúrgicos ambulatóriais.

\section{INTRODUÇÃO}

O número de cirurgias ambulatoriais cresce a cada dia em todo o mundo; somente nos Estados Unidos são anualmente realizados, aproximadamente, 30 milhões destes procedimentos $(7,10,11)$

Em alguns países como a França, a cirurgia ambulatorial foi estruturada e regulamentada no início dos anos 90; nesse país observou-se a partir de 1994, o predomínio das cirurgias ambulatoriais em relação às cirurgias em regime de hospitalização( ${ }^{(5)}$.

As vantagens da realização dos procedimentos em ambiente ambulatorial, com redução dos custos hospitalares e com benefícios para os pacientes, justificam os incentivos financeiros aplicados nesse setor ${ }^{(1,4,6)}$. Apesar destas vantagens, sejam elas sociais e/ou econômicas, ainda há relutância pela maioria dos cirurgiões neste país na indicação dos procedimentos cirúrgicos anorretais em regime ambulatorial; as principais restrições seriam pelo receio das complicações pós-operatórias precoces, possível controle inadequado da dor e pelo valor da remuneração. Em algumas ocasiões a resistência vem do próprio paciente. Com as melhorias das técnicas anestésicas, grande parte das cirurgias, em especial as proctológicas, pode ser realizada com segurança em ambulatório ${ }^{(8,12,13,15,17)}$. O serviço de cirurgia ambulatorial do Hospital das Clínicas da Faculdade de Medicina de Botucatu-HC-FMB, Botucatu, SP., criado em 1996, atende a diversas especialidades (Gastrocirurgia, Urologia, Dermatologia, Oftalmologia, Otorrinolaringologia, Vascular, Cirurgia Pediátrica, Ortopedia, Ginecologia e Cirurgia Plástica), conta com quatro salas cirúrgicas, um enfermeiro e quatro auxiliares de enfermagem. Funciona em dois turnos diários de 4 horas de trabalho em dias úteis. Todo o material é preparado no próprio local.

No presente objetivou-se o estudo dos resultados obtidos com o tratamento cirúrgico ambulatorial, de diversas doenças anorretais, num total de 437 cirurgias, analisando sua eficácia, segurança e complicações.

\section{MÉTODO}

O estudo retrospectivo dos 437 casos foi realizado através da revisão de prontuários dos pacientes atendidos no Serviço de Cirurgia Ambulatorial do HC-FMB, no período de 1996 a 2000.

Os pacientes chegavam ao centro cirúrgico ambulatorial cerca de meia hora antes do procedimento, em jejum de 8 horas, sendo orientados quanto à realização de enema com fosfato de sódio monobásico e dibásico 3 a 4 horas antes do mesmo. Todos foram submetidos a anestesia local ou raquianestesia.

Departamento de Cirurgia e Ortopedia - Disciplina de Gastroenterologia Cirúrgica - Faculdade de Medicina de Botucatu - UNESP

Endereço para correspondência: Prof. Rogério Saad Hossne - Disciplina de Gastroenterologia Cirúrgica - Faculdade de Medicina de Botucatu - 18618-970 - Botucatu, SP. 
Após monitorização eletrocardiográfica, foi realizada punção venosa periférica e iniciada sedação intravenosa com 5 a $7 \mathrm{mg}$ de midazolam diluídos em $10 \mathrm{~mL}$ de solução aquosa. A posição cirúrgica na maioria dos pacientes foi em decúbito ventral com um coxim sob o púbis. A solução anestésica empregada consistiu de lidocaína a $1 \%$, com ou sem vasoconstrictor, sendo realizada a infiltração perianal e submucosa, em leque.

As técnicas cirúrgicas empregadas foram as mesmas utilizadas nos pacientes em regime de internação: doença hemorroidária (técnica aberta de Fergunson); fissura anal (fissurectomia com esfincterotomia interna lateral); nos condilomas (unidade eletrocirúrgica); cisto pilonidal (técnica de incisão e curetagem) e fístula anal (fistulotomia com curetagem).

Após o procedimento cirúrgico, o paciente era mantido na sala de recuperação por cerca de 1 a 2 horas, quando então recebia alta hospitalar em conjunto com as orientações pós-operatórias.

\section{RESULTADOS}

TABELA 1 - Distribuição dos casos por ano. Freqüência absoluta (p) e relativa (f)

\begin{tabular}{ccc}
\hline & $\mathrm{p}$ & $\mathrm{f}$ \\
\hline 1996 & 25 & $5,7 \%$ \\
1997 & 83 & $19 \%$ \\
1998 & 96 & $22 \%$ \\
1999 & 112 & $25,6 \%$ \\
2000 & 121 & $27,7 \%$ \\
\hline
\end{tabular}

TABELA 2 - Distribuição por faixa etária. Freqüência absoluta (p) e relativa $(f)$

\begin{tabular}{lcc}
\hline & $\mathrm{p}$ & $\mathrm{f}$ \\
\hline Até 45 anos & 274 & $62,7 \%$ \\
45-65 anos & 131 & $30 \%$ \\
Maior 65 anos & 32 & $7,3 \%$ \\
\hline
\end{tabular}

TABELA 3 - Distribuição por sexo. Freqüência absoluta (p) e relativa $(f)$

\begin{tabular}{ccc}
\hline & $\mathrm{p}$ & $\mathrm{f}$ \\
\hline Masculino & 192 & $44 \%$ \\
Feminino & 245 & $56 \%$ \\
\hline
\end{tabular}

GRÁFICO 1 - Patologias anorretais. Distribuição da freqüência absoluta (p) e relativa (f)

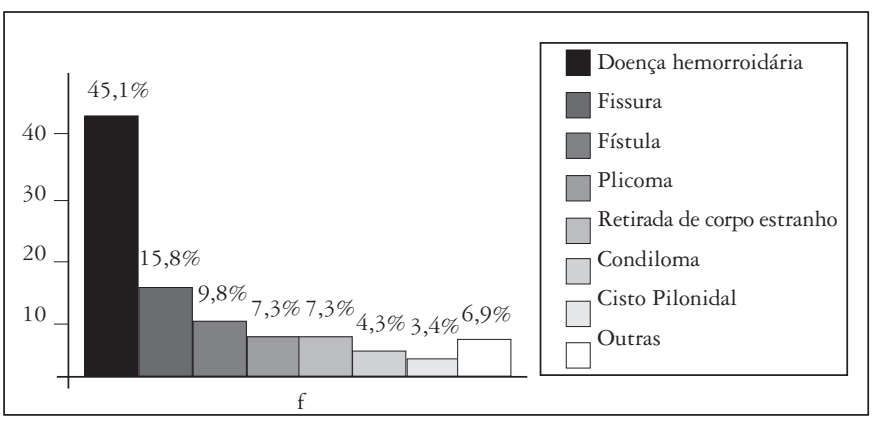

Outras - Trombose hemorroidária, anoplastia, esfincteroplastia, dilatação anal, drenagem de abscesso
TABELA 4 - Tipo de anestesia. Distribuição da freqüência absoluta (p) e relativa $(\mathrm{f})$

\begin{tabular}{ccc}
\hline & $\mathrm{p}$ & $\mathrm{f}$ \\
\hline Local & 423 & $96,8 \%$ \\
Raqui & 14 & $3,2 \%$ \\
\hline
\end{tabular}

TABELA 5 - Tipo de anestésico. Distribuição da freqüência absoluta (p) e relativa $(f)$

\begin{tabular}{ccc}
\hline & $\mathrm{p}$ & $\mathrm{f}$ \\
\hline $\begin{array}{c}\text { Lidocaína sem } \\
\text { vasoconstrictor }\end{array}$ & 371 & $85 \%$ \\
$\begin{array}{c}\text { Lidocaína com } \\
\text { vasoconstrictor }\end{array}$ & 49 & $11,2 \%$ \\
Outros & 17 & $3,8 \%$ \\
\hline
\end{tabular}

GRÁFICO2 - Principais tipos de complicações. Distribuição da freqüência absoluta $(\mathrm{p})$ e relativa $(\mathrm{f})$

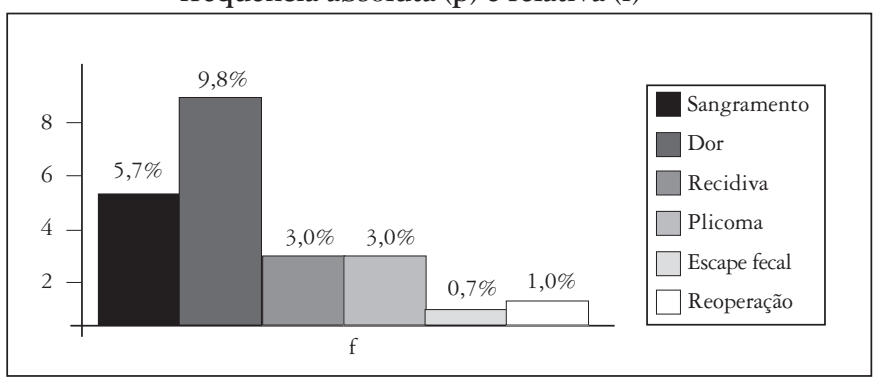

\section{DISCUSSÃO}

É cada vez mais freqüente o tratamento em ambulatório das patologias orificiais em coloproctologia em quase todos os serviços especializados. As técnicas cirúrgicas empregadas, habitualmente, são de fácil e rápida execução, o que contribui para o êxito dos procedimentos em ambulatório.

No caso do HC-FMB, a partir de 1996, com a criação do centro cirúrgico ambulatorial, houve aumento progressivo no número desses procedimentos, conforme demonstrado na Tabela 1, o mesmo relatado por diversos autores, no nosso meio ${ }^{(8,12,14,15)}$.

Atualmente, no HC-FMB adotou-se como norma a realização das cirurgias orificiais anorretais de menor complexidade em ambulatório, em vista da rapidez e agilidade no atendimento dos pacientes, diminuindo desta forma, o tempo de espera para a realização dessas cirurgias; outro fato importante reside na dificuldade de internação de doenças de menor complexidade, em contra-posição a pacientes com doenças neoplásicas, que necessitam de internação prolongada. Essas características dos hospitais universitários na realização de cirurgias de pequeno porte já foi relatada por SOBRADO et al. ${ }^{(16)}$.

Quanto à análise das variáveis estudadas, no que diz respeito a sexo e idade, nota-se que a maioria dos pacientes é jovem $(62,8 \%)$, com menos de 45 anos de idade (Tabela 2), havendo discreta predominância de pacientes do sexo feminino (Tabela 3 ), predomínio este também evidenciado em outros estudos ${ }^{(10,11)}$.

Quanto ao tipo de anestesia, na maioria das vezes, utilizou-se somente a local, sendo a lidocaína sem vasoconstrictor a droga mais utilizada, reservando-se para casos isolados a raquianestesia (Tabelas 4, 5); a anestesia loco-regional com lidocaína é, de fato, a mais freqüentemente utilizada nas cirurgias ambulatoriais, 
chegando a taxas próximas a $90 \%{ }^{(11,17,19)}$. Este tipo de sedação e de anestesia possibilita a realização segura e eficaz desses procedimentos cirúrgicos, bem como permitem o retorno precoce do paciente ao seu domicílio e recuperação mais rápida.

A doença mais prevalente foi a hemorroidária (45,1\%), seguida, em menor número, por fissuras e fistulas $(25,6 \%$ ) (Gráfico 1); esses dados demonstram que essas são as patologias orificiais mais prevalentes em nosso meio, assim como o observado em outros serviços ${ }^{(8,12,16)}$, bem como com os dados referidos na literatura ${ }^{(2,3,9,13,18,20)}$.

A incidência de complicações precoces (dor, sangramento, infecção,etc.) e tardias (plicomas e mamilos residuais, estenose, etc.)

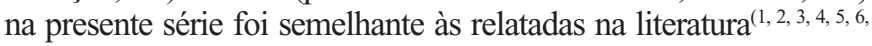
$7,8,9,10,11,12,18,19,20,21)$. O índice geral de complicações precoces nos 437 procedimentos realizados foi de $15,5 \%$, sendo a maioria delas tratada com medidas clínicas. Quanto à retenção urinária, muito freqüente nesse tipo de procedimento, apenas três pacientes evoluíram com a necessidade de cateterização vesical. No serviço onde foi desenvolvido este estudo, não se realizou a antibioticoprofilaxia para tais procedimentos, embora isto seja rotina em alguns serviços ${ }^{(1,2,3,4,5}$, $6,7,8,9,10,11,12,13,14,15,16$, não apresentando nenhuma complicação séptica nos pacientes operados. Quanto aos demais tipos de complicações, verificou-se que a dor pós-operatória foi a complicação mais freqüente (Gráfico 2), sendo que o uso de dipirona, diclofenaco sódico e/ou inibidores da ciclooxigenase foi suficiente para o alívio da dor na maioria dos pacientes desta série. Resultados semelhantes são encontrados na literatura $(10,11,12,13,14,15,16,17,18,19,20,21)$.

Segundo estudo feito por SOBRADO et al. ${ }^{(16)}$ para a estimativa de custo, comparando doentes submetidos a hemorroidectomia em unidade ambulatorial com outros submetidos ao mesmo procedimento em esquema de internação hospitalar, conclui-se que os pacientes operados em ambulatório o custo médio foi de 370 dólares americanos, quando comparado com o de 540, quando o procedimento foi realizado em regime de internação, acarretando uma economia de 31,5\%. No Serviço de Cirurgia Ambulatorial do HC-FMB não foram realizados esses cálculos, mas provavelmente os custos devam ser semelhantes.

\section{CONCLUSÃO}

Os resultados apresentados mostram a boa resolução da cirurgia ambulatorial nas patologias anorretais. Constatou-se que as doenças proctológicas orificiais podem ser tratadas com segurança e baixa morbidade em centro cirúrgico ambulatorial, acarretando diminuição dos custos e agilidade na resolução dos casos. As técnicas cirúrgicas são de fácil execução quando a cirurgia é bem indicada, o nível de complicação é baixo, e a recuperação pós-operatória do paciente se faz em curto espaço de tempo.

Saad-Hoosne R, Prado RG, Bakonyi-Neto A. Outpatient surgery for anorectal diseases: the experience of the Clinics Hospital of the Medical School, Botucatu, SP, Brazil, in 437 cases. Arq Gastroenterol 2005;42(3):136-8.

ABSTRACT - Background - The number of outpatient surgical procedures performed in hospitals, as well as in private clinics, increases daily. In some countries, such as France, outpatient operations outnumber inpatient operations. Objective - To evaluate results obtained at the Surgical Outpatient Clinic of the Clinics Hospital of the Medical School, Botucatu, SP, Brazil. Patients and Methods - A retrospective study of 437 clinical cases of anorectal anomalies, analyzing the variables age bracket, gender, pathology, and postoperative complications. Results - We observed predominance of patients younger than 45 (62.8\%) and of females (56\%). The most common ailment was hemorrhoids (45.1\%) and the most frequent postoperative complications were pain (9.8\%) and bleeding (7.3\%). Conclusions - Our results demonstrate that it is possible to perform various simple procedures related to the treatment of anorectal abnormalities at the outpatient level and therefore at a lower cost. The number of postoperative complications was no higher than that resulting from inpatient surgery.

HEADINGS - Intestine, large, surgery. Ambulatory surgical procedures.

\section{REFERÊNCIAS BIBLIOGRÁFICAS}

1. Fuhrmann J, Grimm H, Schnettler R. [Is ambulatory surgery at a maximum care clinic justified?] Langenbecks Arch Chir Suppl Kongressbol 1997;114:590-3.

2. Gold G. Unanticipated admission to the hospital following ambulatory surgery. JAMA 1989;21:3008-10.

3. Heino T. Results of 500 general surgery patients operated in the ambulatory surgery unit. Ann Chir Gynaecol 1992;81:295-9.

4. Hosteng T, Solheim K. [Ambulatory surgery and incentives]. Tidsske Nor Laegeforen 1996;116:872-3.

5. Johanet H, Mauckey P, Gaux F. Ambulatory surgery. Organization and results. Chirurgie 1997; $122: 35-8$.

6. Junghanns K. [Efficacy and economics of ambulatory surgery in the hospital]. Langenbecks Arch Chir Suppl Kongressbol 1997;114:594-6.

7. Kozak LJ, Owings MF. Ambulatory and inpatient procedures in United States, 1995. Vital Health Stat 1998;13(135):1-116.

8. Medeiros RR. Tratamento ambulatorial das afecções proctológicas. In: Habr-Gama A, Barone B, editores. Atualização em coloproctologia. $44^{\circ}$ Congresso Brasileiro de Coloproctologia. São Paulo: Aquarela; 1995. p.189-91.

9. Nielsen TR, Nielsen BV, Jorgensen PB. [Patients assessment in ambulatory surgery. A questionnaire study]. Ugeskr Laeger 1996;158:1823-6.

10. Owings MF, Kozak LJ. Ambulatory and inpatient procedures in the United States, 1996. Vital Health Stat 1998;13:1-119.

11. Pokras R, Kozak JL, McCarthy E. Ambulatory and inpatient procedures in United States, 1994. Vital Health Stat 1997;3:1-113.
12. Ramos JR, Pinho M, Valory E, Nascimento ML, Souza PCT. Cirurgia ambulatorial em coloproctologia. Rev Bras Coloproctol 1988;8:11-3.

13. Smith LE. Ambulatory surgery for anorectal diseases: an update. South Med J 1986;79:163-6. Sobrado CW, Habr-Gama A. Hook-needle puncture: a new technique of local anesthesia for anorectal surgery. Dis Colon Rectum 1996;39:1330-1.

14. Sobrado CW, Sokol S. Esfincterotomia interna lateral subcutânea em unidade ambulatorial para o tratamento da fissura anal: análise de 265 doentes. Rev Bras Coloproctol 1994;14:235-9.

15. Sobrado CW, Habr-Gama A. Hook-needle puncture. A new technique of local anesthesia for anorectal surgery. Dis Colon Rectum 1996;39:1330-1.

16. Sobrado CW, Nahas SC, Marques CF, Habr-Gama A. Cirurgia ambulatorial sob anestesia local em proctologia: experiência e análise do resultado de 503 operações. Rev bras Coloproctol. 2001;21:228-33.

17. Stern H, McLeod R, Cohen Z, Ross T. Ambulatory procedures in anorectal surgery. Adv Surg 1987;20:217-43

18. Swan BA, Maeslin G, Trabec KB. Symptom distress and functional status changes during the just seven days after ambulatory surgery. Ahesth Analg 1998;86:739-45.

19. Tagliolatto Jr L. Analgesia de condução na cirurgia anorretal. Aspectos técnicos. Rev Bras Coloproctol 1993;13:113-6.

20. Twerky R, Fishman D, Homel P. What happens after discharge? Return hospital visits after ambulatory surgery. Anesth Analg 1997;84:319-24.

21. Weber M, Angermann P, Milkkelsen J, Helkjaer PE, Bjorn Anderden T. Ambulatory surgery and anesthesia. An inquiry study. Ugeskr Laeger 1993;155:2799-803. 